\title{
The Safety of Resistance Training in Children-What Do We Really Know!
}

\author{
Bareket Falk \\ Brock University
}

How many times have you been confronted by the statement that resistance training ${ }^{1}$ is unsafe for growing children, due to the susceptibility of their growing bones or connective tissue to injury? How many times have you tried to explain that it is an unfounded myth?

I have lost count . . . .

In every one of my courses, I strive to dispel this apparent myth. I refer to numerous position or consensus statements of reputable professional organizations $(2,4-6,9)$, which actually advocate for resistance training in childhood. In all of these statements, resistance training is cited as safe for children and adolescents, as it is for mature adults.

But is it? What are those statements based on? To a great extent, they rely on the fact that prospective studies of resistance training in children and adolescents rarely report any such injuries or adverse events. Most reports of resistance-training-related injuries stem from retrospective studies or anecdotal reports. There is a saying that "the plural of anecdote is not data." Actually, it is data. But is it reliable and valid evidence?

A retrospective study that analyzed "weightlifting"-related emergency-room visits between 2002 and 2005 found children to incur less sprains and strains compared with adults. However, children sustained more accidental injuries, due to improper weights and equipment handling (12). The authors reasonably argued that the accidental injuries could be prevented with proper supervision.

The relevant question is not whether there is a risk of injury for children and adolescents in resistance training, or any sport or physical activity for that matter. There is some inherent risk of an adverse event in any physical activity. Given proper supervision, there are two pertinent questions in this regard:

(1) In children and adolescents, is the risk of an adverse event in resistance training greater than in other sports or physical activities?

(2) Is the risk of an adverse event in resistance training greater in children and adolescents than in adults?

There are other related questions. For example, what is the prevalence of mild, moderate, or severe adverse events among children and adolescents engaged in resistance training? Are these adverse events linked to dosage (volume and intensity of training) and are some youth more susceptible than others (eg, those with specific chronic conditions)? And importantly, do any incurred injuries in children and adolescents have long-term adverse consequences?

Falk (bfalk@brocku.ca) is with Brock University, St. Catharines, Ontario, Canada.
To properly address these questions, one needs to examine detailed reports of rates, types, severity, and circumstances of adverse events related to resistance training and other activities, in youth as well as in adults. As a first step, researchers need to report any occurrence of an adverse event related to a resistance training intervention. Such reporting is currently found in a very small proportion of published studies. Most researchers do not report adverse events at all. In a report of resistance-training studies in older adults, Liu and Latham (8) indicated that, of the 121 trials identified, $43 \%$ provided no mention of adverse events. No study I am aware of has performed a similar analysis in children and adolescents. This lack of reporting might reflect the fact that there were no adverse events to report or that minor events were viewed as too benign. Typically, however, researchers do not realize the importance of such reporting and are not aware of the recommended reporting methodologies. Reporting of no adverse events is as important as the reporting of any intervention-related injury. Otherwise, how can resistance training be claimed as safe for youth?

There are various recommendations for the reporting of interventions in general and exercise interventions in particular (see www.equator-network.org for a comprehensive list). These recommendations include the reporting of adverse events. As researchers, we are aware of the importance of detailed description of our experimental design and methods, so that these can be replicated and our results validated. Such reporting is also instrumental for our findings to be translated into practice. An essential part of detailed description ought to be the reporting of any adverse events or their absence. Although a training protocol might be highly effective in increasing strength or other capacity, omitting injured or otherwise adversely affected participants from the results prevent the readers from evaluating the pros and cons of employing such protocol in future research or practical applications.

The Consolidated Standard of Reporting Trials statement was originally developed for the reporting of randomized controlled trials (1) and has subsequently been updated for more specific designs (11). It includes a checklist of 22 items aimed at enhancing reporting quality, one of which is reporting "All important harms or unintended effects in each group." Recently, an exercise-specific reporting template was developed by Slade et al $(14,15)$ to facilitate the interpretation, translation, and implementation of exercise interventions. The 16-item Consensus on Exercise Reporting Template comprises key exercise descriptors including adverse events (ie, "Describe the type and number of adverse events that occurred during exercise").

Detailed reporting is essential for knowledge users who may translate the findings into their own work or practice. It is also essential for researchers who conduct systematic reviews or metaanalysis. Several journals include some reporting checklist in the 
editorial policy (eg, International Journal of Sports Physical Therapy [13]). However, systematic reviews of exercise intervention studies in diverse populations (eg, older population [10], athletes [3]) reveal that current reporting is still inadequate and does not generally adhere to the reporting guidelines.

The guidelines described previously, and most other reporting guidelines, include checklists for authors to ensure that important details and descriptors are provided. However, the effectiveness of such guidelines is dependent on their adoption and use by the research community (10). Hopewell et al (7) found that when journals require authors to review checklists as part of the manuscript submission process, the result is increased quality of information and number of reported items. If we are to refute the myth of resistance training being dangerous for children and adolescents, the reporting of adverse events, or lack thereof in any resistancetraining study, is essential.

\section{Endnote}

${ }^{1}$ For the purpose of this editorial, the term "resistance training" is used to describe training involving work against resistance, aimed at increasing or maintaining muscle strength or function. This is sometimes termed "strength training" and can take the form of weight training, plyometric training, etc.

\section{References}

1. Altman DG, Schulz KF, Moher D, et al. The revised CONSORT statement for reporting randomized trials: explanation and elaboration. Ann Intern Med. 2001;134(8):663-694. PubMed ID: 11304107 doi:10.7326/0003-4819-134-8-200104170-00012

2. American Academy of Pediatrics Council on Sports Medicine and Fitness, McCambridge TM, Stricker PR. Strength training by children and adolescents. Pediatrics. 2008;121(4):835-840. PubMed ID: 18381549 doi:10.1542/peds.2007-3790

3. Charlton PC, Drew MK, Mentiplay BF, Grimaldi A, Clark RA. Exercise interventions for the prevention and treatment of groin pain and injury in athletes: a critical and systematic review. Sports Med. 2017;47(10):2011-2026. PubMed ID: 28497284 doi:10.1007/ s40279-017-0742-y

4. Faigenbaum AD, Kraemer WJ, Blimkie CJ, Jeffreys I, Micheli LJ, Nitka M, Rowland TW. Youth resistance training: updated position statement paper from the national strength and conditioning association. J Strength Cond Res. 2009;23(suppl 5):S60-S79 . doi:10.1519/ JSC.0b013e31819df407
5. Faigenbaum AD, Kraemer WJ, Cahill B, et al. Youth resistance training: position statement paper and literature review. Strength Cond J. 1996;18(6):62-75. doi:10.1519/1073-6840(1996)018<0062: YRTPSP $>2.3 . \mathrm{CO} ; 2$

6. Golan R, Falk B, Hoffman JR, Hochberg Z, Ben-Sira D, Barak Y. Resistance training for children and adolescents. Position statement by the International Federation of Sports Medicine (FIMS). In: Chan KM, Micheli LJ, editors. Sports and Children. Hong Kong: Williams \& Wilkins Asia-Pacific Ltd.; 1998: pp. 265-270.

7. Hopewell S, Ravaud P, Baron G, Boutron I. Effect of editors' implementation of CONSORT guidelines on the reporting of abstracts in high impact medical journals: interrupted time series analysis. BMJ. 2012;344:e4178. PubMed ID: 22730543 doi:10. 1136/bmj.e4178

8. Liu CJ, Latham N. Adverse events reported in progressive resistance strength training trials in older adults: 2 sides of a coin. Arch Phys Med Rehabil. 2010;91(9):1471-1473. PubMed ID: 20801270 doi:10. 1016/j.apmr.2010.06.001

9. Lloyd RS, Faigenbaum AD, Stone MH, et al. Position statement on youth resistance training: the 2014 International Consensus. $\mathrm{Br} \mathrm{J}$ Sports Med.2014;48(7):498-505. PubMed ID: 24055781 doi:10.1136/ bjsports-2013-092952

10. Mack DE, Wilson PM, Santos E, Brooks K. Standards of reporting: the use of CONSORT PRO and CERT in individuals living with osteoporosis. Osteoporos Int. 2018;29(2):305-313. PubMed ID: 28971256 doi:10.1007/s00198-017-4249-Z

11. Moher D, Hopewell S, Schulz KF, et al. CONSORT 2010 Explanation and Elaboration: updated guidelines for reporting parallel group randomised trials. J Clin Epidemiol. 2010;63(8):e1-e37. PubMed ID: 20346624 doi:10.1016/j.jclinepi.2010.03.004

12. Myer GD, Quatman CE, Khoury J, Wall EJ, Hewett TE. Youth versus adult "weightlifting" injuries presenting to United States emergency rooms: accidental versus nonaccidental injury mechanisms. J Strength Cond Res. 2009;23(7):2054-2060. PubMed ID: 19855330 doi:10. 1519/JSC.0b013e3181b86712

13. Page P, Hoogenboom B, Voight M. Improving the reporting of therapeutic exercise interventions in rehabilitation research. Int $J$ Sports Phys Ther. 2017;12(2):297-304. PubMed ID: 28515984

14. Slade SC, Dionne CE, Underwood M, Buchbinder R. Consensus on Exercise Reporting Template (CERT): Explanation and Elaboration Statement. Br J Sports Med. 2016;50(23):1428-1437. PubMed ID: 27707738 doi:10.1136/bjsports-2016-096651

15. Slade SC, Dionne CE, Underwood M, et al. Consensus on Exercise Reporting Template (CERT): Modified Delphi Study. Phys Ther. 2016;96(10):1514-1524. PubMed ID: 27149962 doi:10.2522/ptj. 20150668 